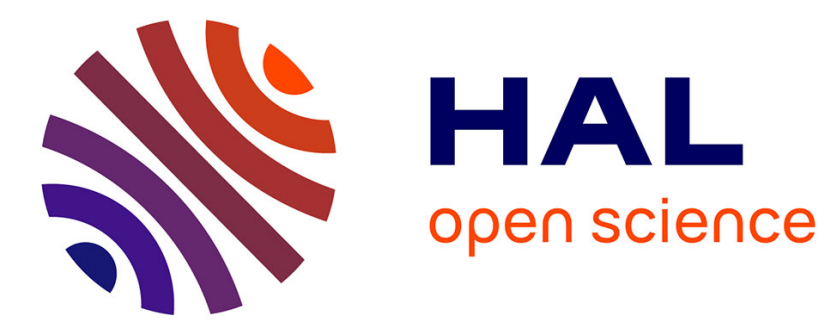

\title{
Finite size effects in cluster-cluster aggregation
}

R.C. Ball, R. Jullien

\section{To cite this version:}

R.C. Ball, R. Jullien. Finite size effects in cluster-cluster aggregation. Journal de Physique Lettres, 1984, 45 (21), pp.1031-1035. 10.1051/jphyslet:0198400450210103100 . jpa-00232446

\section{HAL Id: jpa-00232446 https://hal.science/jpa-00232446}

Submitted on 1 Jan 1984

HAL is a multi-disciplinary open access archive for the deposit and dissemination of scientific research documents, whether they are published or not. The documents may come from teaching and research institutions in France or abroad, or from public or private research centers.
L'archive ouverte pluridisciplinaire HAL, est destinée au dépôt et à la diffusion de documents scientifiques de niveau recherche, publiés ou non, émanant des établissements d'enseignement et de recherche français ou étrangers, des laboratoires publics ou privés. 
Classification

Physics Abstracts

$05.50-64.60$

\title{
Finite size effects in cluster-cluster aggregation
}

\author{
R. C. Ball \\ Cavendish Laboratory, Cambridge CB3 OHE, U.K.
}

and $\cdot \mathbf{R}$. Jullien

Laboratoire de Physique des Solides, Université Paris-Sud, 91405 Orsay Cedex, France

(Reçu le 18 juin 1984, accepté le 4 septembre 1984)

\begin{abstract}
Résumé. - On propose une estimation, à partir de tailles finies, pour la dimension fractale d'amas obtenus au cours d'un processus hiérarchique d'agrégation d'amas. On montre que cette estimation est rigoureusement indépendante de la taille pour $d=1$ et au-dessus de la dimension critique supérieure $d_{\mathrm{c}}$. Pour $1<d<d_{\mathrm{c}}$, elle varie faiblement avec la taille, ce qui conduit à de meilleures valeurs pour les dimensions fractales extrapolées, comme le montrent des résultats numériques, avec trajectoires Browniennes et linéaires, pour $2 \leqslant d \leqslant 5$.
\end{abstract}

\begin{abstract}
A finite size estimate is proposed for the fractal dimension of clusters obtained through hierarchical kinetic cluster-cluster aggregation. This estimate is shown to be exactly size-independent for $d=1$ and above the upper critical dimension $d_{\mathrm{c}}$. For $1<d<d_{\mathrm{c}}$, it varies only weakly with size, leading to better values for the extrapolated fractal dimension, as shown by numerical results on Brownian and linear trajectories for $2 \leqslant d \leqslant 5$.
\end{abstract}

Recently, a model of " kinetic clustering of clusters " $[1,2]$, considering the aggregation of Brownian diffusive clusters, has been proposed to describe flocculation of colloids and aerosols. A particularly simple, hierarchical, formulation of this model has been presented by Botet $e t$ al. [3], rediscovering an old idea by Sutherland and Goodarz-Nia [4]. In this formulation, successive collections of clusters are built iteratively. The clusters of the old collection are grouped into independent pairs. The two clusters of each pair are moved towards each other along a given trajectory (random walk in [3], straight line in [4]) until they touch, forming a cluster of the new collection. Thus, starting from $P_{0}$ individual particles, one generates at step $k, P_{0} / 2^{k}$ clusters of $N=2^{k}$ particles. The radius of gyration of each cluster is defined as :

$$
R_{N}^{2}=\frac{1}{2 N^{2}} \sum_{i, j}\left(\mathbf{r}_{i}-\mathbf{r}_{j}\right)^{2}
$$

where $\mathbf{r}_{i}$ locates the $i$ th particle inside the cluster and where the double sum covers all the cluster sites. Numerically, $R_{N}$ is estimated for size $N$ by averaging $R_{N}^{2}$ over all the clusters of the $k$-genera- 
tion. The fractal dimension $D$ of the clusters, defined by :

$$
R_{N} \sim N^{1 / D^{\prime}} \text { for } N \rightarrow \infty
$$

was estimated, in reference [3], by extrapolating to $N \rightarrow \infty$, an effective $N$-dependent exponent $D_{\text {eff }}(N)$ obtained by comparing two successive steps of the iterations :

$$
D_{\text {eff }}(N)=\ln 2 / \ln \left(R_{2 N} / R_{N}\right) .
$$

This procedure, initially done in $d=2$ dimensions [3], was extended up to $d=5$ [5]. While, for $d=2$, the curve $D_{\text {eff }}(N)$ versus $N^{-1}$ extrapolates quite well, with a negative curvature, when $N^{-1} \rightarrow 0$, for larger $d$ values, $D_{\text {eff }}$ becomes more and more $N$-dependent and, even, a change of curvature occurs around $d \simeq 4$, making difficult to estimate the extrapolated $D$ values. This kind of problem often occurs in finite cell scaling methods, especially when there is no help from exact calculations as to the form of the large- $N$ correction terms.

In this Letter we propose an alternative expression for the effective $N$-dependent fractal dimension :

$$
D^{*}(N)=\ln 4 / \ln \left\{\left(R_{2 N}^{2}-1 / 4\right) / R_{N}^{2}\right\}
$$

Clearly this is consistent with (2), $D^{*} \rightarrow D$ as $N \rightarrow \infty$. We show that $D^{*}(N)$ is rigorously independent of $N$ for $d=1$ as for values of $d$ higher than the above upper critical dimension. Reinterpreting previous numerical results this way, $D^{*}(N)$ is only slightly $N$-dependent for intermediate $d$-values, leading to a better estimation of the extrapolated fractal dimension.

To justify this formula, we consider the "Sutherland's ghost " model, which has been proposed to represent the situation above the upper critical dimension [6]. This is exactly the same model as described above, except that, now, we allow the particles to interpenetrate freely. In this model, when considering the coalescence of clusters $\mathrm{A}$ and $\mathrm{B}$ of $N$ particles into a single cluster of $2 N$ particles, $R_{2 N}$ can be evaluated exactly as a function of $R_{N}$ by calculating the sum :

$$
8 N^{2} R_{2 N}^{2}=\sum_{i, j}\left(\mathbf{r}_{i}-\mathbf{r}_{j}\right)^{2} .
$$

The sum can be divided in four parts according to the fact that $i$ and $j$ can belong to the same old cluster A or B or not. This gives :

$$
8 N^{2} R_{2 N}^{2}=2 N^{2} R_{N}^{2}+2 N^{2} R_{N}^{2}+2 \sum_{i_{\mathrm{A}}, j_{\mathrm{B}}}\left(\mathbf{r}_{i_{\mathrm{A}}}-\mathbf{r}_{j_{\mathrm{B}}}\right)^{2} .
$$

Introducing the sticking points $\mathbf{k}_{\mathrm{A}}$ and $\mathbf{k}_{\mathrm{B}}$, which are separated by one monomer distance $\mathbf{a}$ of unit length $\left(a^{2}=1\right)$, this expression writes :

$$
R_{2 N}^{2}=\frac{1}{2} R_{N}^{2}+\frac{1}{4 N^{2}} \sum_{i_{\mathrm{A}}, j_{\mathrm{B}}}\left(\mathbf{r}_{i_{\mathrm{A}}}-\mathbf{r}_{k_{\mathrm{A}}}+\mathbf{a}+\mathbf{r}_{k_{\mathrm{B}}}-\mathbf{r}_{j_{\mathrm{B}}}\right)^{2} .
$$

It has been shown [6] that, in the Sutherland's ghost model, each point of a cluster has the same probability to be a sticking point and, moreover, the orientation of the connecting link a is completely independent of any distance $\mathbf{r}_{i_{\mathrm{A}}}-\mathbf{r}_{\boldsymbol{k}_{\mathrm{A}}}$ between particles of a given cluster. Thus, when taking the average over all the possible sticking points, one gets :

$$
R_{2 N}^{2}=\frac{3}{2} R_{N}^{2}+\frac{1}{4}
$$


This demonstration has been used [7] to provide an even quicker derivation of the fractal dimension of the ghost model, $D_{\mathrm{g}}=\ln 4 / \ln (3 / 2) \simeq 3.42$ already derived in (6). Moreover, for our purpose here, one can observe that, since the formula is essentially exact for all $N$, the only corrective term, for finite $N$, comes from the $1 / 4$ term in the right hand side of equation (7). Thus, when introducing an effective exponent such as $D^{*}(N)$ defined by formula (3), this exponent is strictly equal to $D_{\mathrm{g}}$, independent of $N$, for the Sutherland's ghost model.

It can be also straightforwardly seen that $D^{*}(N)$ is independent of $N$ for $d=1$ since in that case the exact formula, $R_{N}=\left(N^{2}-1\right) / 12$, leads to :

$$
R_{2 N}^{2}-\frac{1}{4}=\left(4 N^{2}-1\right) / 12-1 / 4=4 R_{N}^{2} \text {. }
$$

Thus, when estimating the effective fractal dimension by $D^{*}(N)$, instead of $D_{\text {eff }}(N)$, it appears that one kills trivial $R_{N}^{-2}$ correction terms which exist both for $d=1$ and above $d_{\mathrm{c}}$. Obviously, for intermediate $d$ values, there must remain other non-trivial correction terms. However the size variation of $D^{*}(N)$ is expected to be considerably smaller than that of $D_{\text {eff }}(N)$. This is effectively the case when analysing the results of numerical computations.

Numerical results for $D^{*}(N)$ as a function of $N^{-1}$ from simulations are shown in figures 1,2 for aggregation by Brownian and linear trajectories, respectively. For Brownian trajectories the results are those already reported in reference [5]. They consider a purely random walk on a $d$-dimensional hypercubic lattice. For $d=2,3,4,20$ trials have been considered, all starting with $P_{0}=4096$ particles and the process has been stopped after 5 iterations, so that $20 \times 128=$ 2560 independent clusters of 32 particles were reached. For $d=5$, only 5 trials were considered. Figure 1 must be compared with figure 2 of reference [3] giving $D_{\text {eff }}(N)$ versus $N^{-1}$. The change of curvature of $D_{\text {eff }}$ can be entirely explained by the trivial $R^{-2}$ corrections. This effect is now suppressed when considering $D^{*}$ which is only slightly varying with $N$. Note that the values obtained when comparing $N=2$ and 4 are already inside the error bars of the extrapolated estimates of $D$ reported in reference [5]. Obviously, the statistical (Monte-Carlo) errors still remain, but this new procedure eliminates the important systematic errors of the previous analysis. The new estimates, with smaller error bars for $d=4,5$, are listed in table I.

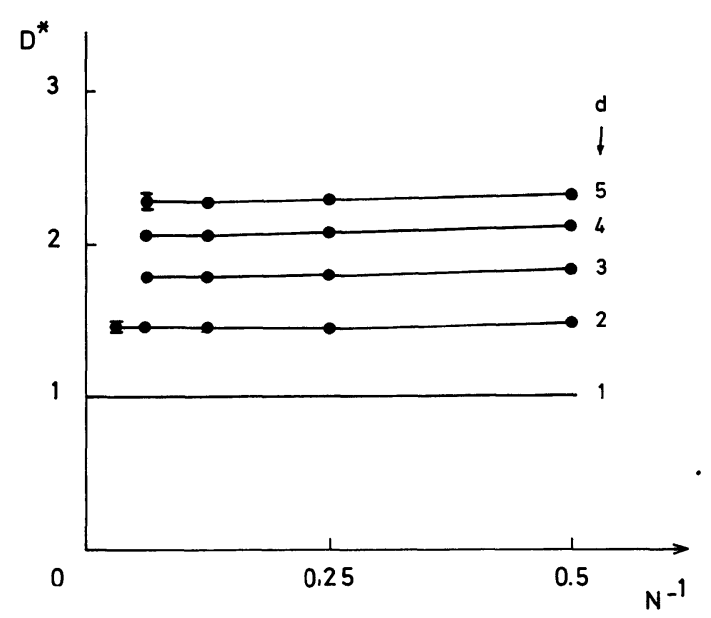

Fig. 1. - Plot of $D^{*}$ versus $N^{-1}$ for dimensions $d=2,3,4,5$ in the case of Brownian trajectories. 


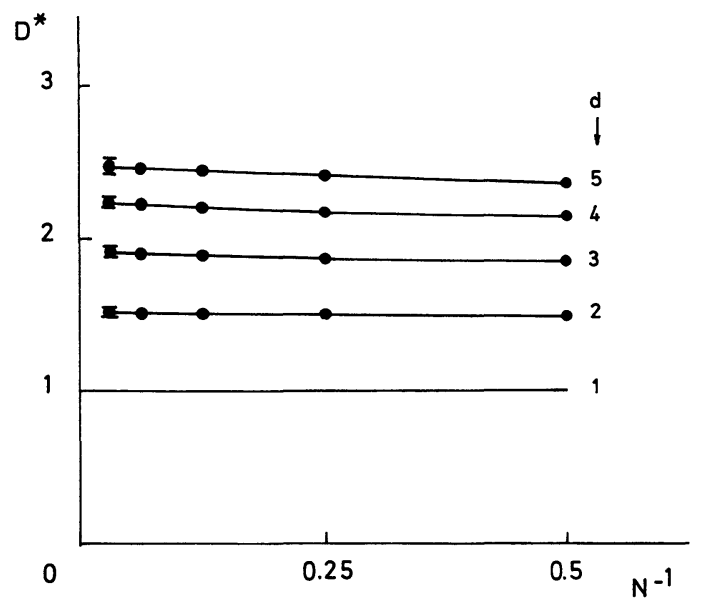

Fig. 2. - Plot of $D^{*}$ versus $N^{-1}$ for dimensions $d=2,3,4,5$ in the case of linear trajectories.

Table I. - Numerical estimates of the fractal dimension of clusters for Brownian $\left(d_{\mathrm{w}}=2\right)$ and linear $\left(d_{\mathrm{w}}=1\right)$ trajectories.

$\begin{array}{ccc}d & d_{\mathrm{w}}=2 & d_{\mathrm{w}}=1 \\ \overline{2} & \overline{-} & - \\ 3 & 1.44 \pm 0.04 & 1.51 \pm 0.03 \\ 4 & 1.78 \pm 0.04 & 1.91 \pm 0.03 \\ 5 & 2.05 \pm 0.05 & 2.22 \pm 0.04 \\ & 2.27 \pm 0.06 & 2.47 \pm 0.05\end{array}$

For linear trajectories, we have considered an off-lattice hierarchical model similar to that recently considered by Meakin [8]. The two clusters of the old generation are now. moved towards each other along an arbitrary straight line in space, until they touch (particles are considered as hard spheres of unit diameter). Here we have considered 1024 trials, starting with $P_{0}=512$ particles, stopping after 6 iterations, so that 8192 independent clusters of 64 particles were reached. The results for the extrapolated fractal dimension are listed in table I. Due to the procedure adopted here the precision of these values compares with those of Meakin [8] who considered considerably larger aggregates, using much more computing time.

In conclusion, we think that, apart from the interest of knowing finite size effects in aggregation of clusters, this study can provide help to those who have only modest computing facilities.

\section{Acknowledgments.}

We would like to acknowledge discussions with R. Botet and M. Kolb. We thank D. Taupin and J. Noguès (Service de Microdensitométrie, CNRS, Orsay) for help in computing. 


\section{References}

[1] Meakin, P., Phys. Rev. Lett. 51 (1983) 1119.

[2] Kolb, M., Botet, R. and Jullien, R., Phys. Rev. Lett. 51 (1983) 1123.

[3] Botet, R., Jullien, R. and Kolb, M., J. Phys. A 17 (1984) L75.

[4] Sutherland, D. N. and Goodarz-Nia, I., Chem. Eng. Sci. 26 (1971) 2071.

[5] Jullien, R., Kolb, M. and Botet, R., J. Physique Lett. 45 (1984) L-211.

[6] Ball, R. C. and Witten, T. A., Proceedings of the third Conference on Fractals, Gaitherburg, Md., Nov. 21-23, 83. To be published in J. Stat. Phys.

[7] Ball, R. C. and Witten, T. A., unpublished.

[8] Meakin, P., Phys. Rev. A 29 (1984) 997. 